\title{
INSERÇÃO DO BIOMÉDICO NA ÁREA DA IMAGENOLOGIA EM HOSPITAIS E CLÍNICAS NO RIO GRANDE DO SUL
}

\author{
THE INSERTION OF BIOMEDICAL SCIENTISTS IN THE IMAGING AREA OF \\ HOSPITALS AND CLINICS IN RIO GRANDE DO SUL
}

Guilherme da Costa, Fernanda Rocha da Trindade, Luís Felipe Pissaia, Arlete Eli Kunz da Costa

Centro Universitário Univates

\begin{abstract}
Biomedical Scientists in the imaging area could work in health teams, complementary diagnostic activities, as well as perform radiological exams for which they are legally qualified. The objective of this study was to evaluate the insertion of biomedical professionals in the imaging area of hospitals and clinics in Rio Grande do Sul, Brazil. It is a descriptive, exploratory study of a qualitative approach that was developed by means of interviews with six biomedical scientists. It was ascertained the importance of disciplines in the imaging area for future professionals, as well as internships in the area. Some difficulties were identified, especially with regards to the employability of those professionals, as well within as the graduation scope, due to the lack of offering of specific disciplines in imaging and a drop in the quality of its contents, which has a direct impact on the qualification of biomedical scientists.
\end{abstract}

Key words: Health Personnel; Radiography; Patient Assistance Team.

\section{Resumo}

O biomédico na área da Imagenologia pode atuar em equipes de saúde, em atividades complementares de diagnóstico, bem como realizar exames radiológicos para os quais esteja legalmente habilitado. O objetivo deste estudo é avaliar a inserção do profissional biomédico na área da Imagenologia em hospitais e clínicas do Rio Grande do Sul, Brasil. Trata-se de um estudo descritivo e exploratório com abordagem qualitativa, desenvolvido por meio de entrevista a seis biomédicos. Verificou-se a importância da realização de disciplinas na área de Imagenologia para os futuros profissionais, bem como a realização de estágios na área. Identificaram-se algumas dificuldades principalmente na empregabilidade destes profissionais, bem como no âmbito da graduação devido ao déficit na oferta de disciplinas específicas em Imagenologia e queda na qualidade de seus conteúdos que impactam diretamente na qualificação do biomédico.

Palavras-chaves: Pessoal de Saúde; Radiografia; Equipe de Assistência ao Paciente. 


\section{Introdução}

A presença de novos profissionais na área da saúde é de extrema importância na área do radiodiagnóstico e da radioterapia, chamada de Imagenologia, não é diferente ${ }^{1}$. Através das diversas modalidades de exames de Imagenologia, é feito o diagnóstico por imagem, que consiste no estudo dos órgãos e sistemas do corpo humano ${ }^{2}$.

O profissional biomédico está presente no radiodiagnóstico, principalmente na Tomografia Computadorizada (TC), na Ressonância Magnética (RM) e na Medicina Nuclear (MN) ${ }^{1}$. Já na área referente a tratamento, o biomédico pode atuar na radioterapia, executando o tratamento prescrito pelo radioterapeuta, e na dosimetria, associado com os radioterapeutas e os físicos médicos ${ }^{1,2,3}$. Ele realiza todo e qualquer tipo de exame na área de imagem, incluindo o pós-processamento da mesma, neste sentido estudos nessa área podem promover o desenvolvimento profissional e assistencial ${ }^{1}$.

$\mathrm{Na}$ TC, o biomédico pode operar equipamentos, criar e definir protocolos de exames, administrar os meios de contraste, realizar entrevista com o paciente e fazer análise prévia dos dados para fins específicos da atividade. Na RM, além das funções citadas anteriormente, pode atuar nas áreas de RM Funcional e Espectroscopia por RM. Na MN, pode operar equipamentos como o SPECT, PET/CT e $\mathrm{PET} / \mathrm{RM}$, realizar estudos "in vivo" e "in vitro" $\mathrm{e}$ auxiliar $O$ médico nos procedimentos terapêuticos ${ }^{2}$.

No final da década de 90, já existiam profissionais habilitados e especializados na área de Imagenologia, pois vários hospitais e empresas perceberam que os Biomédicos poderiam ter uma formação diferenciada e atuar como multiplicadores nas equipes às quais pertenciam ${ }^{1}$. Em São Paulo, por exemplo, os profissionais biomédicos na área da Imagenologia estão inseridos no mercado de trabalho desde a mesma década ${ }^{3}$.

Isso acabou contribuindo para que a habilitação em Imagenologia se tornasse uma das especializações atraentes aos alunos dos cursos de Biomedicina ${ }^{1}$. Por essa razão, o biomédico, com atuação nessa área, assim como todos os profissionais, devem estar comprometidos e motivados, além de estar ciente da demanda de conhecimento teórico e científico que a área exigirá dele e de sua equipe ${ }^{4}$. Portanto, considera-se que o cuidado do profissional amplia o vínculo com o indivíduo, a família e a sociedade, contribuindo para ações de promoção, prevenção e proteção à saúde, trazendo, assim, muitos benefícios à população ${ }^{5}$.

O profissional torna-se importante na equipe, visto que ele pode estabelecer metas e avaliar as melhores formas de realizar um exame de imagem, assim como proporcionar à equipe a execução de medidas padronizadas que facilitem o processo de trabalho ${ }^{3}$. Considera-se que sua inserção nos serviços de saúde deve ser documentada e estudada com o intuito de agregar credibilidade ao seu trabalho, além de fomentar pesquisas que contribuam para a área da saúde ${ }^{2}$.

Neste sentido, o objetivo deste estudo, foi avaliar a inserção do biomédico na área de Imagenologia em hospitais e clínicas do Rio Grande do Sul, Brasil.

\section{Metodologia}

Este estudo é descritivo e exploratório, com abordagem qualitativa. A amostra foi composta por seis biomédicos, com registro na 5o Região do Conselho Regional de Biomedicina (CRBM), com habilitação em Imagenologia e que atuam em hospitais e/ou clínicas no Rio Grande do Sul, Brasil.

Com base nos dados dos profissionais registrados no CRBM citado encaminharam-se emails para 40 profissionais, sendo que destes 34 não responderam e apenas seis retornaram a mensagem colocando-se a disposição do pesquisador, totalizando o número de participantes. O e-mail enviado continha a descrição dos objetivos da pesquisa, bem como os critérios éticos que foram preconizados pelo grupo de pesquisadores, sendo ainda repassados os contatos dos mesmos para retorno e confirmação das informações. Uma vez aceito o convite, foram agendadas as entrevistas conforme disponibilidade do profissional e em local de sua preferência, que garantisse a confidencialidade dos dados, garantindo um diálogo tranquilo entre o pesquisador e participante.

A coleta de dados ocorreu entre outubro e novembro de 2016 por meio de entrevista onde se utilizaram questões objetivas e abertas, desenvolvidas pelos pesquisadores. Foram visitadas quatro cidades (Lajeado, Esteio, ljuí e Marau), totalizando 930 quilômetros percorridos. O questionário foi constituído por questões de caracterização dos participantes referentes à idade, sexo, ano de formação e meses 
trabalhando em Imagenologia. Já as questões abertas abarcaram a inserção do profissional biomédico na área da Imagem e no ambiente de trabalho, a exames de imagem realizados por biomédicos, as dificuldades de inserção no mercado de imagem e a habilidades e competências na formação de biomédico para atuar na área.

As entrevistas individuais tiveram duração entre 20 minutos a 1 hora e seus áudios gravados, os quais foram posteriormente transcritos e analisados por pontos focais compatíveis, conforme é preconizado pela Análise de Conteúdo de Bardin ${ }^{6}$. Os nomes dos participantes foram mantidos em sigilo, utilizando-se o codinome "Bmd", seguido de números arábicos sorteados aleatoriamente pelos pesquisadores, buscando-se identificar suas falas na composição dos resultados.

Este estudo foi direcionado pelos preceitos que norteiam a realização de pesquisas com seres humanos, citando a Resolução 466/12 do Conselho Nacional de Saúde. O projeto de pesquisa foi aprovado pelo Comitê de Ética em Pesquisa (COEP) do Centro Universitário UNIVATES, por meio da CAAE $\mathrm{n}$ 응 58371416.2.0000.5310.

\section{Resultados e discussão}

Por meio dos resultados caracterizaram-se os participantes como sendo maioria do sexo feminino, com 83,3\%, com idade entre 26 a 35 anos (média de $28,7 \pm 3,8$ anos). Já o período de trabalho como profissional biomédico na área de Imagenologia variou de menos de um ano para três indivíduos e a mais de quatro anos para os outros 3 participantes, e o ano de formação dos profissionais variou de 2009 a 2016. Neste sentido, os participantes deste estudo caracterizam-se como jovens e se formaram há menos de 10 anos.

Em relação aos cursos de pós-graduação, $50 \%$ dos participantes realizaram um curso referente à Biologia Molecular e Celular, Imagenologia e Estética. Em relação aos exames que mais realizam na área da imagem, quatro profissionais responderam que o de coluna é o mais realizado, seguido dos exames de crânio, joelho, abdômen e tórax. Um participante realiza apenas exames de coluna e de crânio. E outro participante atua no pós-processamento e, por essa razão, não realiza os exames. Quanto a realização da disciplina de Imagenologia durante a graduação apenas um participante $(16,7 \%)$ não cursou disciplina específica. A maioria dos participantes (83,3\%) cursou e avaliou como importante para sua formação e atuação na área.

Segundo os entrevistados a realização da disciplina específica supracitada os qualificou como profissionais, mas, mesmo assim, foi difícil sua inserção no mercado de trabalho, conforme se verificou que, para $83,3 \%$, ela ocorreu de forma negativa, sendo uma experiência de dificuldades.

Os primeiros biomédicos na área de Imagenologia tiveram oportunidade em hospitais que haviam investido milhões de dólares em equipamentos de imagem e não existiam profissionais capacitados para operar essas máquinas ${ }^{1}$. Anos depois, foi instituído o curso de Tecnologia em Radiologia Médica, o que acabou melhorando a qualidade da mão de obra. Ao mesmo tempo, alguns cursos de Biomedicina instituíram a separação da Imagenologia da disciplina de Biofísica, pois acreditaram que fosse facilitar a compreensão dos estudantes ${ }^{7}$.

Conforme Guedes et al. ${ }^{8}$ no campo da prática biomédica, vários problemas tornam-se imediatamente aparentes: a insatisfação de outros profissionais diretamente envolvidos na inserção dos biomédicos; os custos crescentes dos exames; a formação de recursos humanos; o mercantilismo e a competição entre os próprios profissionais da área; além da precariedade dos programas de saúde. Assim, um questionamento sobre o conhecimento e a prática do biomédico contemporâneo parece necessário para poder visualizar quais as possibilidades e desafios dessa ciência quanto à sua relação com o crescimento da autonomia dos sujeitos no processo de saúde e doença ${ }^{9}$.

Em relação aos exames de imagem realizados por biomédicos, verificou-se que os entrevistados acreditam na qualidade dos exames e na garantia de maior segurança para o paciente. Os participantes relataram a importância do biomédico em relação à segurança do paciente, à realização adequada da anamnese, à criação de novos protocolos, às orientações aos familiares, entre outros aspectos, devido ao maior conhecimento teórico sobre o assunto.

Em relação a este conhecimento segue o relato: "Biomédicos possuem conhecimento anatômico e funcional, bem como entendimento laboratorial, que guia muitas vezes a realização do exame." (Bmd 4). Ainda complementa-se na fala a seguir "Possui conhecimento nas áreas relacionadas à física, anatomia, fisiologia, etc $[\ldots]^{\prime \prime}$. (Bmd 5) 
Aos biomédicos cabe demonstrar o que a lei estabelece, fazendo cumprir a vontade do legislador que the atribuiu, entre suas competências, a realização de serviços de radiografia - excluída a interpretação -, bem como a atuação - sob supervisão médica - em serviços radiodiagnósticos ${ }^{2}$.

Em relação à inserção do biomédico no ambiente de trabalho, $66,7 \%$ dos profissionais em Imagenologia realizaram estágio curricular obrigatório durante a graduação, e 33,3\% já trabalhavam na instituição na qual foram convidados para trabalhar depois de diplomados.

A escolha do local do estágio tornou-se importante para os estudantes, como mostra o relato: "Creio que me inseri adequadamente, devido ao estágio final ter sido realizado nesta empresa e por crer que o tenho feito de maneira satisfatória, assim mostrando que o estágio pode abrir portas." (Bmd 3). Já para outro participante a situação repetiu-se, conforme o relato: "Através de indicação, logo após fazer estágio." (Bmd 6) e no caso de outro profissional houveram dificuldades, conforme a fala: "Através da persistência ao ligar para o RH da empresa. Realizei estágio obrigatório, passei para o curricular e logo em seguida fui contratado". (Bmd 4).

Em relação às habilidades e competências na formação de biomédico para atuar na área, os entrevistados relataram falta da parte prática das disciplinas cursadas de Imagenologia, conforme o relato: "Somente tive contato com a Imagenologia nas aulas (disciplinas conteúdo teórico). Mas a teoria foi sempre bem abordada em relação á física, segurança ao paciente, meios de contraste." (Bmd 1). Outro participante comentou: "Após a disciplina de biofísica, quando aprendi sobre o funcionamento e aplicabilidade dos métodos diagnósticos, tomei conhecimento da possibilidade de me inserir no mercado de trabalho e comecei a buscar oportunidades". (Bmd 4). Neste mesmo sentido comentou-se no seguinte relato que: "Através da disciplina Introdução em Biomedicina fiquei ciente das habilitações e me interessei pela imagem". (Bmd 4).

Em estudos desenvolvidos na área de atuação do biomédico constatou-se que há um déficit nas competências exigidas nas denominadas áreas da imagem, possuindo como base principalmente a falta de disponibilidade de disciplinas específicas nos cursos de graduação $^{2,7,10}$. Ainda compreende-se que a necessidade de qualificação do ensino torna-se urgente sob o limiar de formação dos profissionais na área.

\section{Conclusões}

Considera-se que no estudo houve uma limitação da pesquisa referente ao número de biomédicos que atuam na área de Imagenologia, tornando o número de participantes baixo. Por outro lado, considerando-se o número de biomédicos atuando em Imagenologia, cerca de 40 profissionais no Rio Grande do Sul, torna-se alto, visto tratar-se de uma área nova e em expansão.

Por meio deste estudo, verificou-se a importância da realização de disciplinas específicas na área da Imagenologia nos cursos de graduação, como forma de aperfeiçoamento para os futuros profissionais. A experiência nesta área enquanto estágio também se mostrou como um diferencial no mercado de trabalho, possibilitando a contextualização entre a teoria e a prática. Percebeu-se que a qualificação da prática clínica do biomédico atrela-se à preparação acadêmica e à busca por conhecimento teórico durante e após a realização de sua graduação.

Por fim, torna-se perceptível a importância dos campos de estágios curriculares dos cursos de graduação como meio de inserção dos profissionais no mercado de trabalho na área da Imagenologia. No entanto, a busca por práticas acadêmicas específicas, como o trabalho com a imagem, demanda uma aprendizagem que deve estar prevista nas grades curriculares oferecidas, fato que se verificou não ocorrer em alguns cursos de Biomedicina. Sendo assim, torna-se importante a escolha do estudante pelo local de estágio, pois este define a habilitação de biomédico na área de Imagenologia.

\section{Referências}

1. Del Campo $M$, Arancibia $S$, Nova $E$, Salazar F, González A, Moltedo B, loannes P, Ferreira J, Manuben A, Becker MI. Hemocianinas, una herramienta inmunológica de la biomedicina actual Hemocyanins as immunostimulants. Rev Med Chile. 2011; 139(2): 236-246. http://dx.doi.org/10.4067/S003498872011000200015

2. Dias HS, Lima LD, Teixeira M. A trajetória da política nacional de reorientação da formação profissional em saúde no SUS. Ciênc. saúde coletiva. $\quad$ 2013; $18(6)$. http://dx.doi.org/10.1590/S1413- 
81232013000600013

3. Gonçalves VD. Caracterização da dose em pacientes devido á produção de imagens de raio-x utilizadas em radioterapia guiada por imagem -IMRT. 2012. Dissertação (Mestrado em Ciências na Área de tecnologia Nuclear Reatores) - Instituto de pesquisas Energéticas e Nucleares/Universidade de São Paulo. São Paulo.

4. Barros JA. Pensando o processo saúde doença: a que responde o modelo biomédico?. Saúde e Sociedade. 2002; 11(1): 67-84.

5. Menéndez E. Modelos de atención de los padecimientos: de exclusiones teóricas y articulaciones prácticas. Ciência \& Saúde Coletiva. 2003; 8(1): 185-207.

6. Bardin L. Análise de conteúdo. São Paulo, 2011.

7. Tonetto AM, Gomes WB. A prática do psicólogo hospitalar em equipe multidisciplinar. Estudos de Psicologia. 2007; 24(1): 89-98.

8. Guedes RC, Nogueira IM, Kenneth RC. Subjectivity as anomaly: epistemological contributions for a criticism of the biomedical model. Ciência \& Saúde Coletiva. 2006; 11(4): 1093-1103.

9. Tesser CD. Social medicalization: biomedical limits and proposals for primary care clinics. Interface - Comunic., Saúde, Educ. 2006; 10(20): 347-62.

10. Costa FB, Trindade MAN, Pereira MLT. A inserção do biomédico no programa de saúde da família. Revista Eletrônica Novo Enfoque. 2010; 11(11): 27-33.

\section{Endereço para Correspondência}

Centro Universitário Univates

e-mail: Ipissaia@universo.univates.br

Recebido em 24/04/2017

Aprovado em 14/07/2017

Publicado em 21/08/2017 